Periodica Polytechnica Architecture, 49(1), pp. 72-76, 2018

\title{
Rome and Its Domes: Drawing, Art and Architecture
}

\author{
Emanuela Chiavoni1* \\ 1 Department of History, Drawing and Restoration, Faculty of Architecture, Sapienza University of Rome, \\ Piazzale Aldo Moro 5., 00185 Roma, Italy \\ *Corresponding author, e-mail: emanuela.chiavoni@uniroma1.it
}

Received: 14 December 2017, Accepted: 23 March 2018, Published online: 04 May 2018

\begin{abstract}
Any analysis performed to gather data about architectural and urban heritage always begins with the observation of reality and the involvement established between the observer and the space around them. A spectator inevitably interprets what they see in a personal manner so as to give objects a specific meaning. The ability to understand the data and the relationship between the parts and then assign it a value varies from person to person. That the mind can mediate the mental processes of perception-actionthought means that any direct analysis to understand a cultural asset is scientifically extremely important. The critical process sparked by an on-site study exploits the concise graphic transcription of what is visible, including the graphics tool used to achieve that representation. This contribution will present a study performed by the author to gather data using the drawings of several domes in the historical centre of the city of Rome.
\end{abstract}

Keywords

drawing, art, architecture, Rome, domes

\section{Introduction}

On-location drawing of built heritage is like no other knowledge-gathering procedure. Our whole body is totally and utterly involved in this delicate process of interpretation and analysis: sight, touch, olfactory perception - all our senses contribute to understanding the reality around us. Freehand drawings enable us to choose and select the most significant features of an architectural work, i. e., the characteristic elements that not only make the artefact unique and recognisable as a whole but also create its cultural identity. On-location drawing plays a key role in the interpretation of forms, structures and space as well as in the comprehension of the decorative elements and details that complete and embellish buildings. When based on direct analysis, all the various graphic interpretations spark an in-depth understanding of the complex urban scenario.

\section{Domes in Rome}

Rome's skyline is multifaceted, varied and inhomogeneous. Although filled with towers and multi-storey buildings of all different shapes and sizes, it is Rome's rounded domes that stand out and make the skyline recognisable the world over. These precious architectures, cited by famous poets and writers and painted by renowned artists throughout history, still attract international tourism. So, what does the skyline of the city of Rome look like today?

The old city centre is full of public and private buildings with unique roofs: churches with all kinds of geometric-shaped domes; bell-towers, each individual in shape and height; geometric towers, elegant and at times even imposing. The force and refinement of these architectures establish urban scenarios, turning them not only into directional signs and visual references for local inhabitants but also into very recognisable historical urban landmarks for tourists passing through the eternal city.

We look at most buildings from the ground as we walk along the streets and through the squares; only by going to the highest points in the city is it possible to get a good, panoramic view, for example, from the Janiculum Hill, Monte Mario or the Monteverde district. Designers throughout the ages have always focused primarily on the way architecture is connected to the earth, the way it is "fixed to the ground", i. e., the shift from the natural (earth) to the artificial (building). In the author's opinion, however, we have never dedicated sufficient study to the way buildings are "fixed to the $s k y$ "; the empty space that creates the backdrop behind the crowning elements of the buildings such as roofing, domes, bell-towers, and roofs. In drawings, this backdrop coincides 


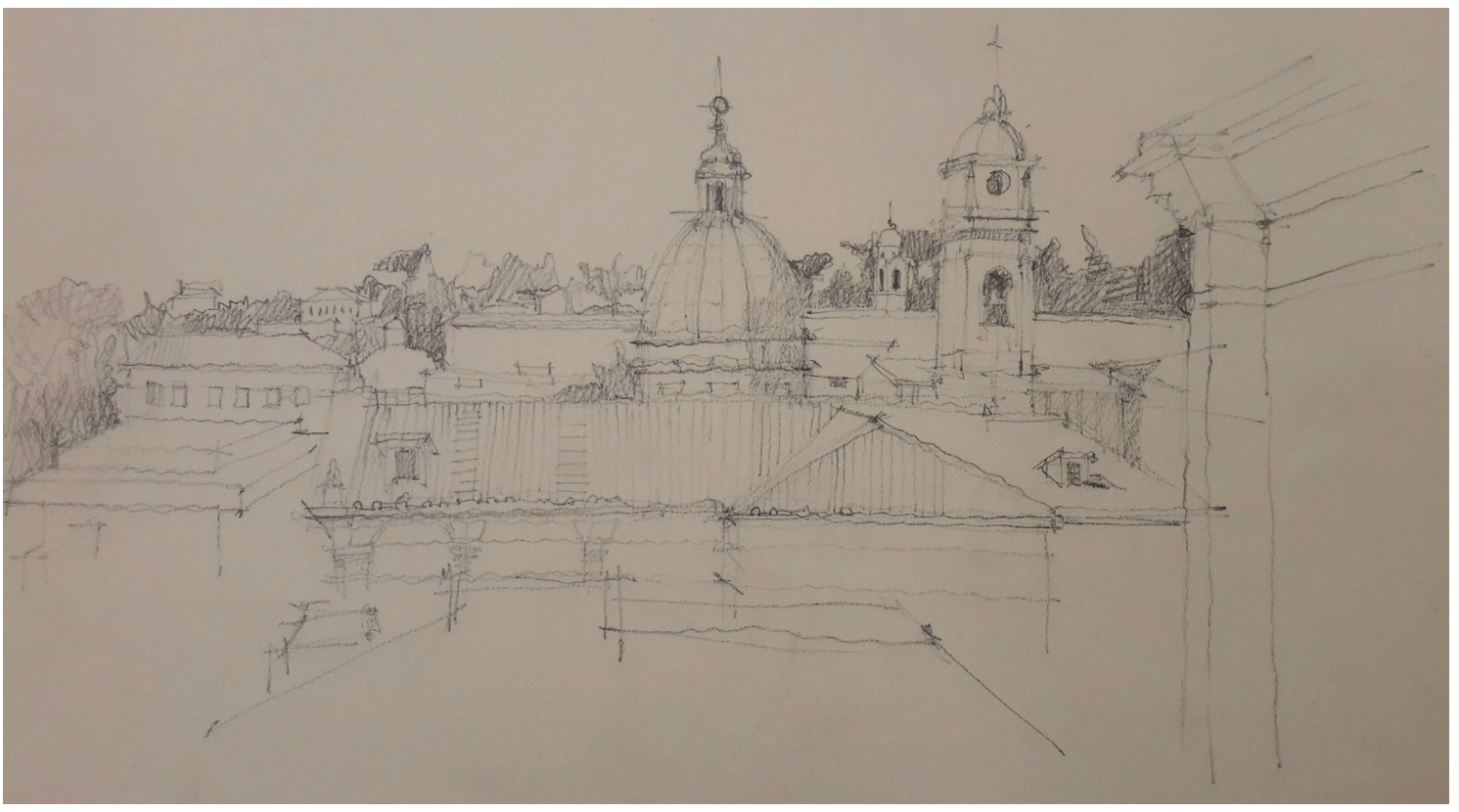

Fig. 1 Roman skyline with the dome of San Rocco Church in the middle. Pencil drawing.

with the top part of the sheet of paper. As a result, in our research, the focus was more on what is called, in the graphical analysis process performed during drawing, the study of how a building is "fixed to the ground" (cit.).

This involves the lines that define the buildings and bring them to a close; the lines characterising the city, which clarify the contours and can be seen, often, only in the distance. The skyline stands out against an ever-changing background; continuous chromatic variations are caused by the seasons, the atmosphere, the weather and the natural rhythms of the sun and shadows throughout the day. These factors change unceasingly, and this is what makes the study of what we can call an apparent contour so interesting. Sometimes, the colour of the sky is similar to the colour of the material covering the domes (for example, a grey-silver hue), thereby making the perimeter of the dome disappear. In fact, in some cases, it's only possible to vaguely see its perimeter through the upper lantern, built in a different material. Similarly, at sunset, the (brick-red) colour of a roof can be confused with a reddish streak in the sky, becoming invisible. At that precise moment, the building looks as if it has no crowning.

\section{Using drawings as an interpretation tool}

Several domes in Rome were interpreted in situ, while a number of drawings were made looking upwards from the ground; several panoramic views of Rome were executed from a distance.
The aim was to draw the domes the way citizens see them, that is, the way they are seen from different locations in the city. The paper concentrated only on these extremely elegant artistic structures, almost in isolation from the buildings on which they rest. The intention was to understand their forms, bulk, volume and the decorations reminiscent of different periods in time. Instead of analysing the way they are fixed to the ground, the focus concentrated exclusively on the upper parts that make up the topmost structures of buildings.

We used real-life representation as an important, selective exercise in direct knowledge and exploited it to monitor and analyse these extremely valuable and important architectural assets with a view to their future enhancement. Studying and examining these complex volumes is always an important, ongoing activity because it allows an understanding of the past and rethink the new. We tried to gather more data about the relationship between each structure and the urban context, building technique, forms, geometries and proportions. The obvious contrast between the curved and rectilinear elements is a very important feature, which we tried to capture and understand with our own eyes. These elegant structures remind us of many designers and historical periods, and drawings help us to gather extensive data not visible to the naked eye. They are always the tool of choice to understand reality and record the harmony intrinsic in these artistic structures since they mediate between what is observed and what really exists. 


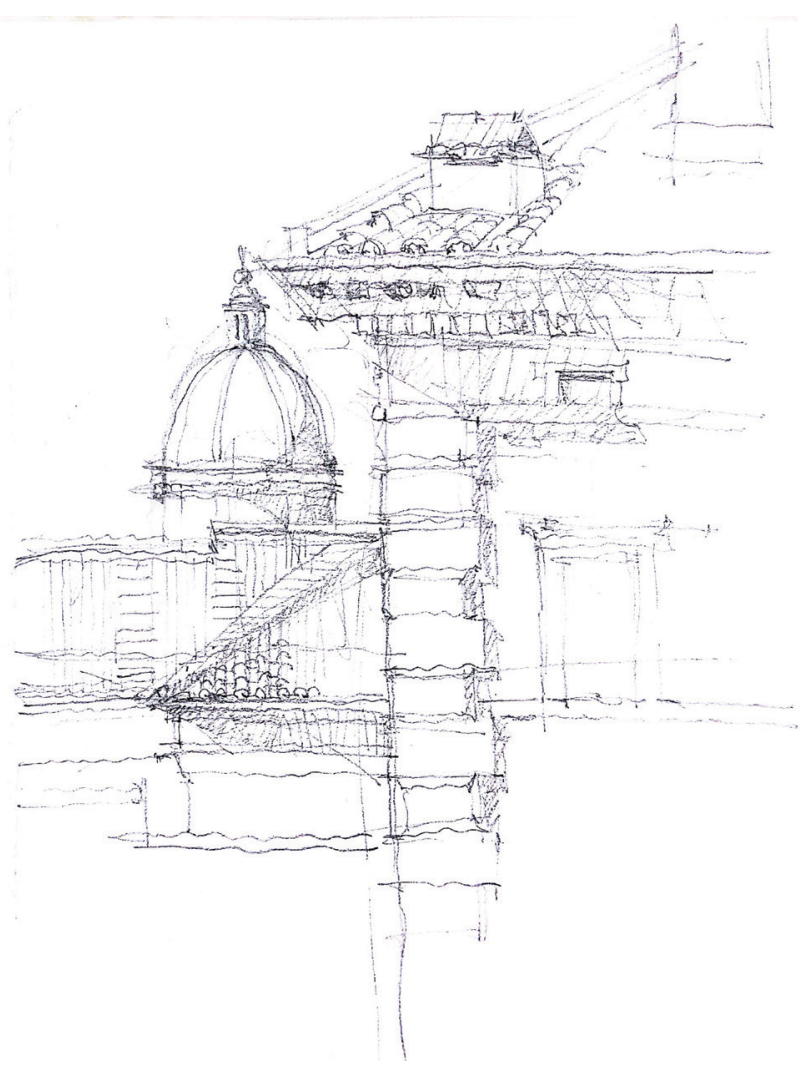

Fig. 2 A detail of the San Rocco Church with Palazzo Borghese in the foreground. Pencil drawing.

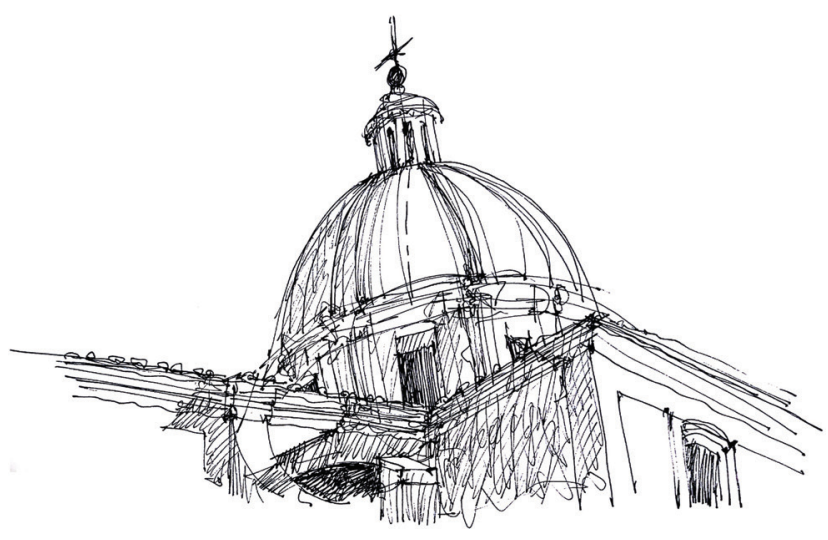

Fig. 3 A close view of the dome of San Rocco Church looking upwards from the ground. Pen and ink drawing.

\section{Representing colours}

"... Unlike numbers, objectively present in what we see around us, colours are subjective entities of perception ... created by the relationship between object and object; the use of colour thus becomes more pondered and significant"

((cit. A. Bonito Oliva in Si fa presto a dire colore))

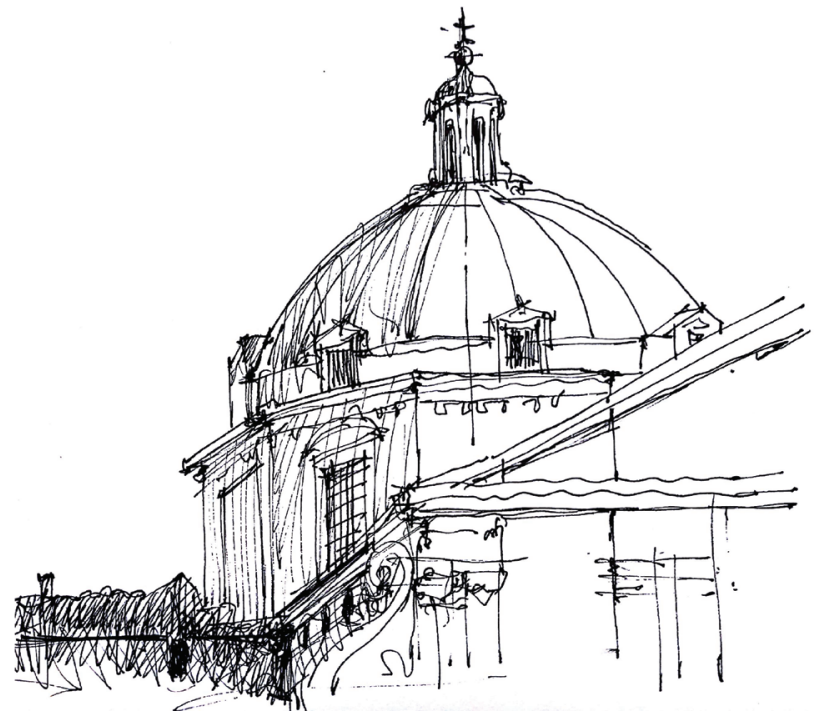

Fig. 4 A study of the geometrical form and proportion of a church dome. Pen and ink drawing.

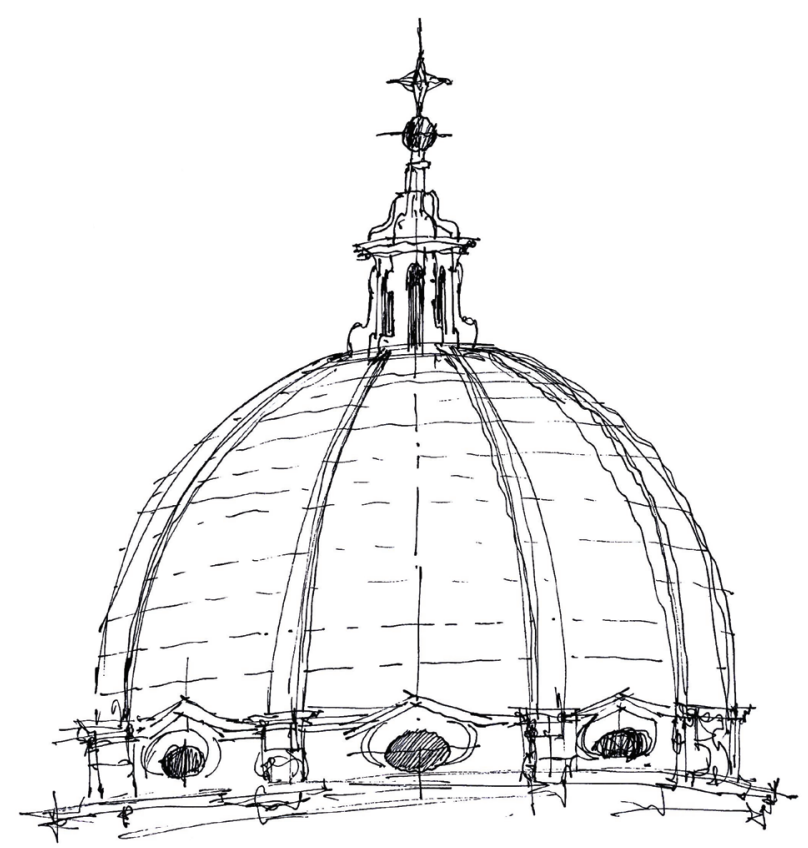

Fig. 5 A close view of the dome of San Girolamo Church looking upwards from the ground. Pen and ink drawing.

In the chromatic study of the domes, watercolour was used to capture the explosive and discontinuous force of colour and the way in which it is variously perceived. This artistic technique, with its intense, forceful expressiveness, is the best medium with which to describe what surrounds us, to interpret transparencies and vibrations and recreate the atmospheres and sensations that are difficult to achieve 


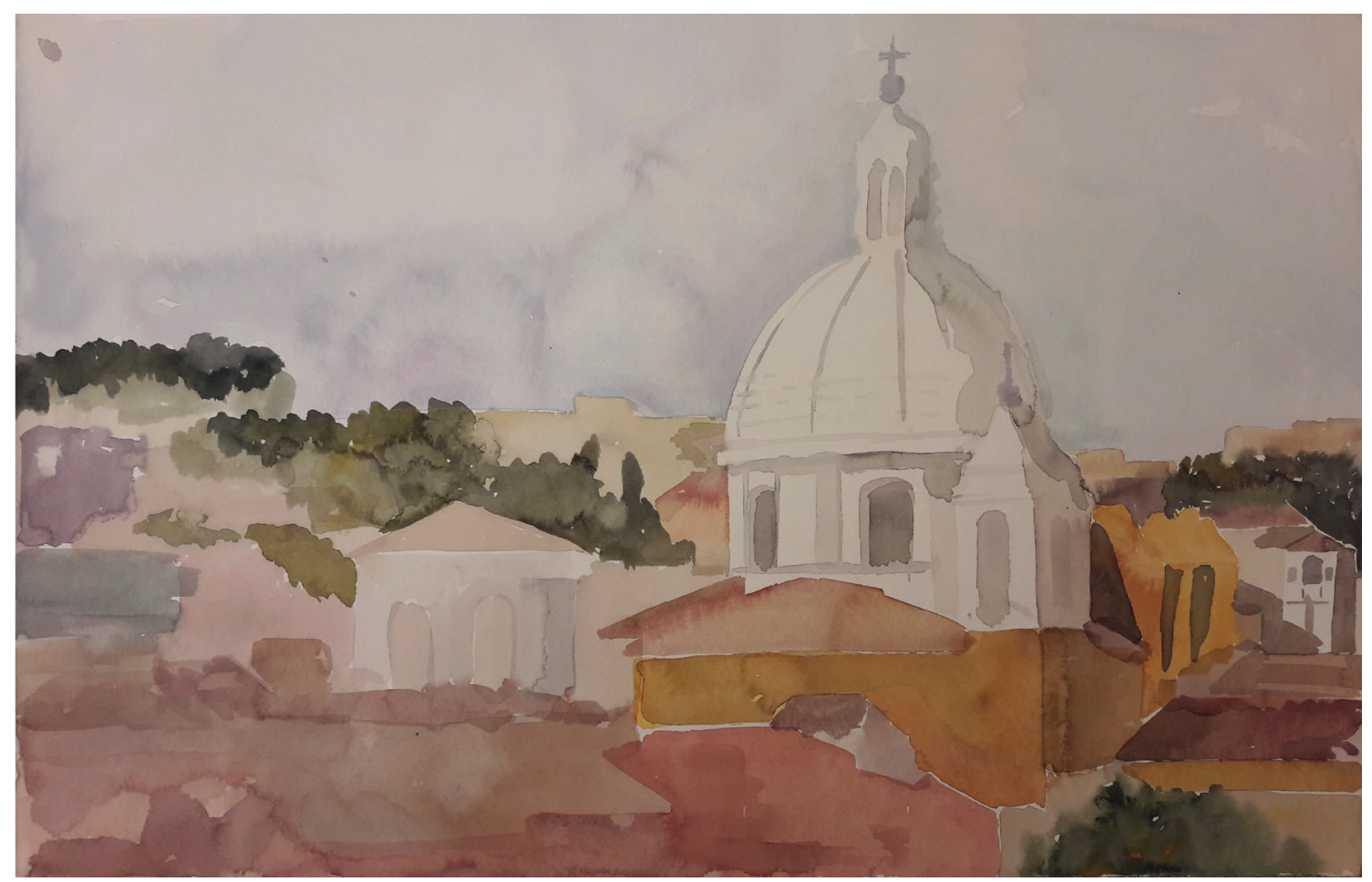

Fig. 6 A skyline of the centre of Rome with the dome of San Rocco Church. Watercolour drawing.

with other graphic techniques. When executing a real-life drawing, the very first visual impact captures the intensity of the light and colour; only afterwards does one appreciate the form, bulk and shape of the structure in question. The graphic process elaborates visual notes, sketches and studio drawings, which gradually help to define volumes, spaces and geometries. It is very important to be both sensitive and critical in order to appreciate the more immaterial, intangible features that characterise a place and make it recognisable.

Drawing the sky on a sheet of paper also plays an important role because it acts as a backdrop to the outline of the dome and contrasts with the colour of the materials; the sky is almost never drawn in black and white representations.

\section{Survey of the domes}

Until around thirty years ago, surveying domes was difficult because the tools needed to survey complex volumes analytically were not available. The exterior could only be surveyed visually (cit. Marinucci) while it was possible to survey the interior of domes by building very high scaffolding to acquire points on the inner surface directly. Instead, in the eighties, it was possible to use terrestrial photogrammetry to study domes scientifically. This procedure was based on a very precise acquisition project; it enabled and still enables us (even if it is very seldom used these days) to exploit specific analytical plotters to recreate, in a laboratory, the stereoscopic situation that existed when the photographs were taken. Apart from the immediate opportunity to observe and interpret domes from above, either directly on Google Earth or specific aerophotogrammetric images, laser scanners currently allow us to acquire data. Using suitable topographic tools, it is now possible to perform very accurate surveys of both the interior and exterior of buildings. This research aims to emphasise how important it is to survey these complex volumes (domes). A survey project must choose both the appropriate methods and the tools required to scientifically establish the geometric and spatial elements of each artefact. Direct, on-location drawing plays a crucial role in this regard; in fact, it is the graphic basis for not only all the operations involved but also the organisation of the work phases.

\section{Conclusions}

Domes are, for many reasons, an important historical feature of the city of Rome, permanent urban signs that provide directions, adorn the urban skyline, and constitute a unique, one-of-a-kind cultural asset that needs to be understood and enhanced. The objective of this contribution is to reinterpret the past using direct graphical operations to rediscover not only the original design but also tackle the history of construction. We executed straightforward, clean, black and white drawings to become familiar with these 
precious, elegant volumes, and painted watercolours to try and record the immaterial and most attractive elements of the atmosphere around the urban spaces where the domes are located. In recording the immaterial, to represent the

\section{References}

Arnheim, R. (1978) "Arte e percezione visiva", (Art and Visual Perception) Feltrinelli, Milano. (in Italian).

Chiavoni, E., De Carlo, L. (2015) "Scorci urbani. Le cupole di Roma nell'opera di Angelo Marinucci", (Urban Views. The Domes of Rome in Works by Angelo Marinucci) Disegnare, Idee, Immagini, 25(51), pp. 56-57. (in Italian).

Chiavoni, E., Docci, M. (2014) "Visione, pensiero, disegni: gli insegnamenti di Francis D. K. Ching | Vision, ideas, drawings, teachings by Francis D. K. Ching", Disegnare, Idee, Immagini, 24(49), pp. 48-59. (in two languages: Italian and English).

Chiavoni, E. (2014) "Drawings on paper. Digital historical archives of the former Radaar Department at the University Sapienza School of Architecture in Rome", SCIRES-IT, 4(2), pp. 117-126.

Chiavoni, E., Fabbri, L., Porfiri, F., Tacchi, G. L. (2012). "La linea del cielo. Sperimentazioni sulla rappresentazione del colore di skyline romani", (The Skyline. Experiments with the Representation of Colour of Roman Skylines) In: Convegno Colore e Colorimetria. Contributi Multidisciplinari, Vol. 8, Maggioli Editore, pp. 673677. (in Italian) light when it falls on the curves is a continuous challenge. The light, as the main element, that dialogues with space and creates shadows, which in turn are such a key element and yet extremely difficult to draw given their fluidity.

Ching, F. D. K. (1990) "Drawing A Creative Process", John Wiley \& Sons, Inc., New York, USA.

Ching, F. D. K., Juroszek, S. P. (1998) "Design Drawing", John Wiley \& Sons, Inc., New York, USA.

De Carlo, L., Chiavoni, E. (2012) "Geometry and symbols in Sant'Ivo alla Sapienza in Rome", In: Proceedings of International Congress Domes in the World, Nardini Editore, Firenze, Italy, pp. 1-10.

De Carlo, L., Chiavoni, E., Romor, J., Wahbeh, W. (2014) "Geometry and Symbols in Sant'Ivo alla Sapienza in Rome", Domes and Cupolas, An International Journal for Architecture, Engineering, Conservation and Culture, Angelo Pontecorboli Editore, Firenze, Italy. 1(1), pp. 65-75.

Docci, M., Chiavoni, E. (2017) "Saper leggere l'architettura", (How to Interpret Architecture) Gius. Laterza \& Figli S. p. A., Bari-Roma, Italy. pp. 1-212. (in Italian).

Morlacchi, M. (2008) "Il disegno a mano libera, il disegno dal vero", (Freehand Drawing, On-location Drawing) In: Il libro del disegno, Gangemi Editore, Roma, Italy. pp.37-69. (in Italian). 